\title{
Subpectoral and Precapsular Implant Repositioning Technique: Correction of Capsular Contracture and Implant Malposition
}

\author{
Hong Ki Lee $\cdot$ Ung Sik Jin $\cdot$ Yoon Ho Lee
}

Received: 8 December 2010/Accepted: 18 March 2011/Published online: 22 April 2011

(C) The Author(s) 2011. This article is published with open access at Springerlink.com

\begin{abstract}
Background Although capsule formation is a naturalhealing process following breast augmentation using implants, a contracted capsule around a poorly positioned implant can act as an obstacle during the corrective procedure to reposition the implant. The ideal treatment of capsular contracture is removal of the capsule and covering the implant with a healthy envelope without scar tissue. However, total capsulectomy in the submuscular space may be difficult, especially if the capsule is firmly attached to the chest wall. This situation may require a highly skilled technique because aggressive capsulectomy could injure the intercostal muscles and vasculature and cause further complications such as pneumothorax. Therefore, the authors have developed a new, less traumatic method of leaving the capsule behind the new implant.

Method From February 2001 through February 2009, the authors treated 74 patients (139 breasts) using a subpectoral, precapsular implant repositioning technique. These patients suffered from capsular contracture or implant malposition after submuscular breast augmentation. The technique is composed of three parts. First, a plane was developed between the anterior wall of the capsule and the posterior surface of the pectoralis major muscle using a periareolar or inframammary approach. After removing the previous implant, the anterior wall of the capsule was fully released from the posterior surface of the pectoralis major
\end{abstract}

H. K. Lee $(\square)$

Image Plastic Surgery Clinic, Seoul, Republic of Korea

e-mail: lee@imageclinic.co.kr

U. S. Jin · Y. H. Lee

Seoul National University Hospital, Seoul, Republic of Korea

e-mail: usj1011@snu.ac.kr muscle and fixed to the posterior wall of the capsule which adhered to the chest wall. The new implant was inserted into the developed subpectoral space, anterior to the capsule.

Results The mean age of the patients was 31 years $($ range $=24-52)$ and the time between the primary and the secondary augmentation was 42 months (range $=4$ months to 12 years). The range for follow-up was from 12 months to 5 years. Median follow-up was 26 months. Postoperative complications included two cases of hematoma but no cases of infection, muscle distortion, or double-bubble deformity. Conclusion This technique is a valid alternative treatment for capsular contracture or malpositioned implant after breast augmentation surgery. It may be less traumatic than the conventional method of total capsulectomy. In addition, this technique reduces the relapse rate of capsular contracture significantly compared to a partial capsulectomy or capsulotomy as the new implant is inserted into a scar tissue-free environment. Good aesthetic results and patient satisfaction was achieved using this method. In our experience, this novel technique is a good alternative method of correcting complications of submuscular implant augmentation.

Keywords Secondary breast augmentation - Capsular contracture $\cdot$ Implant malposition

Breast augmentation is one of the most popular cosmetic surgery procedure. Most of these cases have a high rate of patient satisfaction. However, some women undergo revision to their implants for various reasons. One of eight women with breast implants will have more than one surgery on her breasts [1]. Major reasons for further operations include capsular contracture and improvement of implant position and breast shape. 
Capsule formation around an implant is a result of an immune reaction to the foreign body; the natural response of any living tissue to a foreign object, even inert material. Since the early days of breast augmentation, severe capsular contracture has remained a leading reason for a secondary operation. This complication compromises aesthetic results and causes pain and discomfort to the patient, requiring further treatment or implant removal. The Baker system has remained the gold standard for the classification of capsular contractures. In general, grades I and II are acceptable, whereas grades III and IV usually require surgical intervention. The ideal treatment for capsular contracture is the removal of the capsule along with covering the implant with a healthy envelope without scar tissue. The most effective treatment for capsular contracture grades III and IV is usually implant removal followed by total capsulectomy and reimplantation. However, a total capsulectomy in the submuscular space could be difficult, especially if the capsule is tightly fixed to the chest wall. It may also lead to damage of the surrounding tissue [2]. Aggressive attempts to completely excise in these cases may lead to injury of the intercostal muscle, cause a pneumothorax, or produce major bleeding from intercostal vasculature, which in turn may cause another capsular contracture. Therefore, we have developed a less traumatic and more useful method that leaves the capsule behind the new implant instead of carrying out a total capsulectomy. Another option is to use the prepectoral (subglandular) space for the insertion of the new implant. However, in patients with a relatively small amount of breast tissue or thin skin, this method may achieve suboptimal cosmetic results (the breast shape may be unnatural or the implant may be visible under the skin). In these patients, use of the submuscular plane is a more desirable option. Our technique is also useful in cases of implant malposition. Traditionally, external compression using taping or garments, bolster fixation, percutaneous suture, and internal capsulorrhaphy have been used to treat implant malposition. However, these methods were often insufficient and showed a high recurrence rate [3].

This report describes a novel method for treating capsular contracture and implant malposition. This technique is based on studies that showed that the retained capsules could be resorbed by the body or transformed into fibrous tissue following removal of the implant if there was no inflammation or calcification [4]. It is expected that this technique may be effectively utilized for a secondary operation after submuscular breast augmentation.

\section{Materials and Methods}

Over an 8-year period between February 2001 and February 2009, 74 patients (139 breasts) with capsular contracture or implant malposition post submuscular breast augmentation were treated using the surgical technique described below. Fifty-one patients had capsular contractures and 23 patients had implant malposition. Sixty-five cases were bilateral and nine cases were unilateral. Of the cases of capsular contracture, grade III accounted for 32 and the remaining 19 were grade IV. The 23 cases of implant malposition included 5 cases of bottoming out, 1 case of double-bubble deformity, 15 cases of upward migration, and 2 cases of symmastia. Of the 74 patients, 47 had had primary breast augmentation using silicone implants and 27 had saline implants. Four types of implants were used in the corrective operation over the 8-year period: saline smooth implants were used in 28 patients, saline textured implants in 4, cohesive gel-type silicone smooth implants in 18, and cohesive gel-type silicone textured implants in 24 patients.

\section{Surgical Technique}

Before the operation, preoperative marking was done with the patient standing with arms at rest, facing forward. The midline, current inframammary fold line, planned inframammary fold line, the boundary of the pocket to be dissected, and the incision line were marked. In the cases of capsular contracture, the dissection boundary followed the implant size and profile, degree of glandular ptosis, tissue elasticity of breast parenchyma, and skin. In the cases of implant malposition, the dissection pocket was more meticulously designed to meet an adequate new implant position.

With the patient under general anesthesia and arms abducted to $90^{\circ}$, the marked incision line was infiltrated with a local anesthetic solution (1\% lidocaine mixed with epinephrine 1:200,000). Tumescent solution was carefully injected into the target region in the subglandular plane so that the implant was not punctured. Afterwards, the incision was made on the planned inframammary fold line.

The boundary of dissection followed the preoperative design. In cases of capsular contracture, adequate dissection can be measured according to implant size, profile, and ptosis of glandular tissue. In cases of upward implant malposition, adequate lowering of the inframammary fold line in the subpectoral and subglandular planes is required. In cases of bottoming out and double-bubble deformity cases, the amount of the dissection needs to correspond to an appropriate line of the new inframammary fold.

After the incision was made, subcutaneous tissue was dissected down to the deep fascia; the subglandular plane was also dissected. The upper limit of the subglandular dissection area was set using the degree of glandular ptosis according to the dual-plane augmentation principle by Tebbetts [5]. The free margin of the pectoralis major 
muscle was dissected from the capsule. At this time, a subpectoral dissection was performed between the posterior surface of the pectoralis major muscle and the anterior surface of the anterior wall of the capsule. Thus, the new virgin plane-subpectoral and anterior to the anterior wall of the capsule-could be created for a new implant (Fig. 1).

After confirming that the entire anterior surface of the capsule can be seen, a central incision was made in the anterior surface of the capsule to remove the existing implant. The internal surface of the capsule was well irrigated using normal saline and a betadine solution. The anterior and posterior walls of the capsule were tightly sutured with three or nine stitches to avoid separation. This procedure is critical to avoid seroma formation and downward migration of the new implant in the upright position postoperatively. However, in cases of upward implant malposition, the tight suturing between the anterior wall and the posterior wall of the capsule is not necessary. The peripheral edge of the capsule, especially the inferior half, was sometimes thick, probably due to the accumulation of fluid and hematoma after the initial operation. The thick, band-like scar tissue at the periphery should be carefully and meticulously dissected and removed. If any
Fig. 1 Diagram of new subpectoral, anterocapsular implant repositioning technique. Top left Preoperative lateral view. Top right Dissection between pectoralis major muscle and anterior surface of capsule via inframammary incision. Bottom left Implant removal and tucking of anterior capsule to the posterior capsule and chest wall. Bottom right New implant insertion above previous capsule in dual-plane pocket
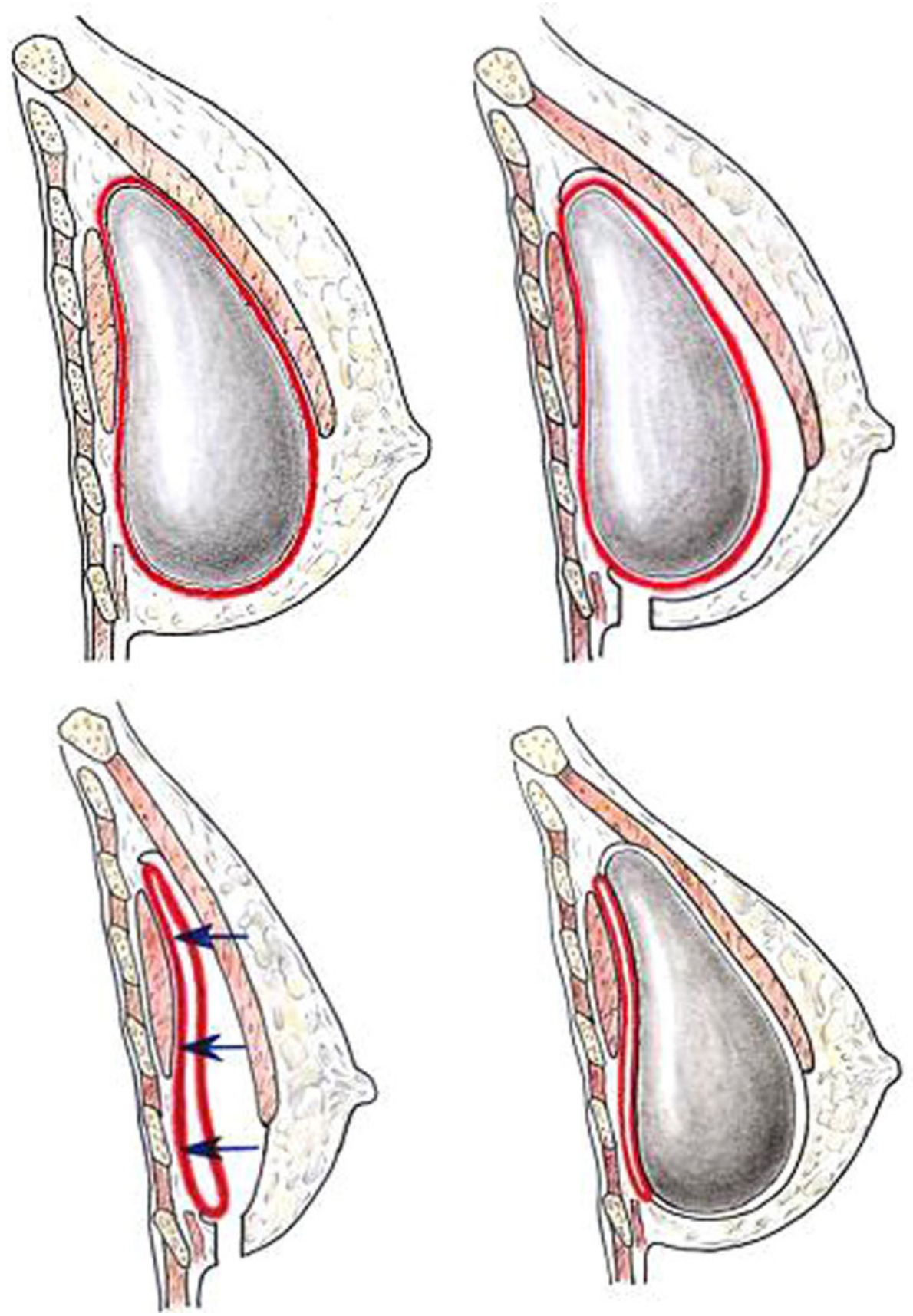
scar tissue remains on the subglandular and subpectoral planes, it could prohibit covering of the implant with a scar tissue-free envelope, which can result in double bubbledeformity or a constriction band at that point.

To prevent a seroma or reactive fluid collection caused by remaining free spaces of the capsule, a small incision at the dependent portion of the anterior capsule was made for drainage. After achieving hemostasis using electrocauterization, the developed plane for the new implant was irrigated using normal saline and betadine solution and the new implant was inserted. A negative suction drain was inserted and the incision was closed. The wound was dressed with compression bandaging around the thorax. The drain was removed 1 or 2 days after the operation and the patient was discharged with a specially made tight support bra (Make Up Bra ${ }^{\circledR}$, VM21 Co., Seoul, Korea) (Fig. 2, bottom photo). If the shell of the new breast implant was smooth, the displacement exercise was applied one week after the operation four times a day for 2 or 3 months. However, if the shell of the new breast implant was textured, no displacement exercise was recommended.

\section{Results}

The average age of the patients was 31 years (range $=24-52$ years) and the average volume of the implants used was $270 \mathrm{~cm}^{3}$ (range $=175-350 \mathrm{~cm}^{3}$ ). The amount of time between the primary mammaplasty and the corrective operation varied, ranging from 4 months to 12 years (mean $=42$ months). The follow-up period ranged from 12 months to 5 years (mean $=26$ months). Two cases of hematoma were observed, but there were no other complications such as seroma formation or infections. The capsular contracture Baker class III recurred in three cases (Figs. 2, 3, 4). The 54 patients who underwent the operation from October 2002 were prescribed leukotriene antagonists for 3 months following surgery [6].

\section{Discussion}

Implant-based augmentation mammaplasty has developed continuously since its introduction in the 1960s, in both the technique and the implants used. The resulting shape, feel, and consistency of the implant are key to a successful operation. Therefore, preventing capsular contracture is of the utmost importance. Although there are many ways to reduce the risk of capsular contractures, it is not always possible to prevent them as capsular contracture is induced by various patient and local breast factors [7, 8] that are not easily controlled. Prevention is the best way of treating capsular contractures, but the rate of their development has not decreased despite continual efforts to achieve this. In addition, a capsule that results from the natural-healing process complicates the correction of implant malposition. Thus, it is very important to learn how to deal with a formed capsule in a secondary operation.

Closed capsulotomy has been used extensively in the past for the treatment of established capsular contracture and was especially popular from the late 1970s through the 1980s [9]. Today, however, closed capsulotomy is rarely performed because of its frequent association with complications such as extracapsular rupture, implant displacement, hematoma formation, and uneven capsule release [10]. In addition, a high recurrence rate of the capsular contracture has also been reported [11]. As a consequence, closed capsulotomy no longer has a role in the management of capsular contracture, and the FDA has declared this procedure contraindicated. Open capsulotomy, or scoring of the capsule without removing it, is similarly unsuccessful and has a contracture recurrence rate of over 50\% [11]. It is also difficult to make an accurately expected pocket with an open capsulotomy procedure. Situations where an open capsulotomy is a reasonable treatment of choice include correction of a malpositioned implant when the breast is soft but the implant is misplaced due to a technical error in formation of the pocket. However, it can bring about unwanted results because of unequal capsule tension. Therefore, due to their complications and high recurrence rates, open and closed capsulotomies are not recommended as effective treatments.

The most effective treatment for capsular contracture, therefore, is total capsulectomy and the insertion of a new implant, but this is a difficult procedure, especially in cases of submuscular augmentation. A thick capsule adherent to the chest wall can be very difficult to excise. Aggressive attempts to totally excise such a capsule may injure intercostal muscle, cause a pneumothorax, or produce major blood loss. Partial capsulectomy, which is excision of the anterior segment of the capsule, is easier and safer than total capsulectomy, but the recurrence rate is higher [2]. The recurrence rates following capsulotomy and partial capsulectomy are higher than that of total capsulectomy because there are no environmental changes surrounding the implant, and the remaining capsule may act as a nidus for the development of another capsular contracture. If the anterior capsule is excised and the posterior surface of the capsule remains, the newly inserted implant will be in contact with the posterior wall of the capsule which is problematic tissue, i.e., biofilm [12] by subclinical bacterial inoculation, and, thus, capsular contracture is more likely to occur.

The technique presented in this report makes it possible to easily dissect and create conditions similar to total capsulectomy in a novel, scar tissue-free environment. Without disturbing the posterior aspect of the capsule adjoining the chest wall, the anterior surface of the capsule 
Fig. 2 A 33-year-old woman. Left column Two years after breast augmentation. The patient suffered from capsular contracture. Right column Ten months after revision augmentation mammaplasty with subpectoral, precapsular implant repositioning technique. Bottom Postoperative garment (Makeup bra ${ }^{\circledR}$ ). Note that we can form the inframammary crease easily and rapidly and can achieve lower-pole expansion effectively because the upper band compresses the breast mound and the lower band restrains the inframammary crease
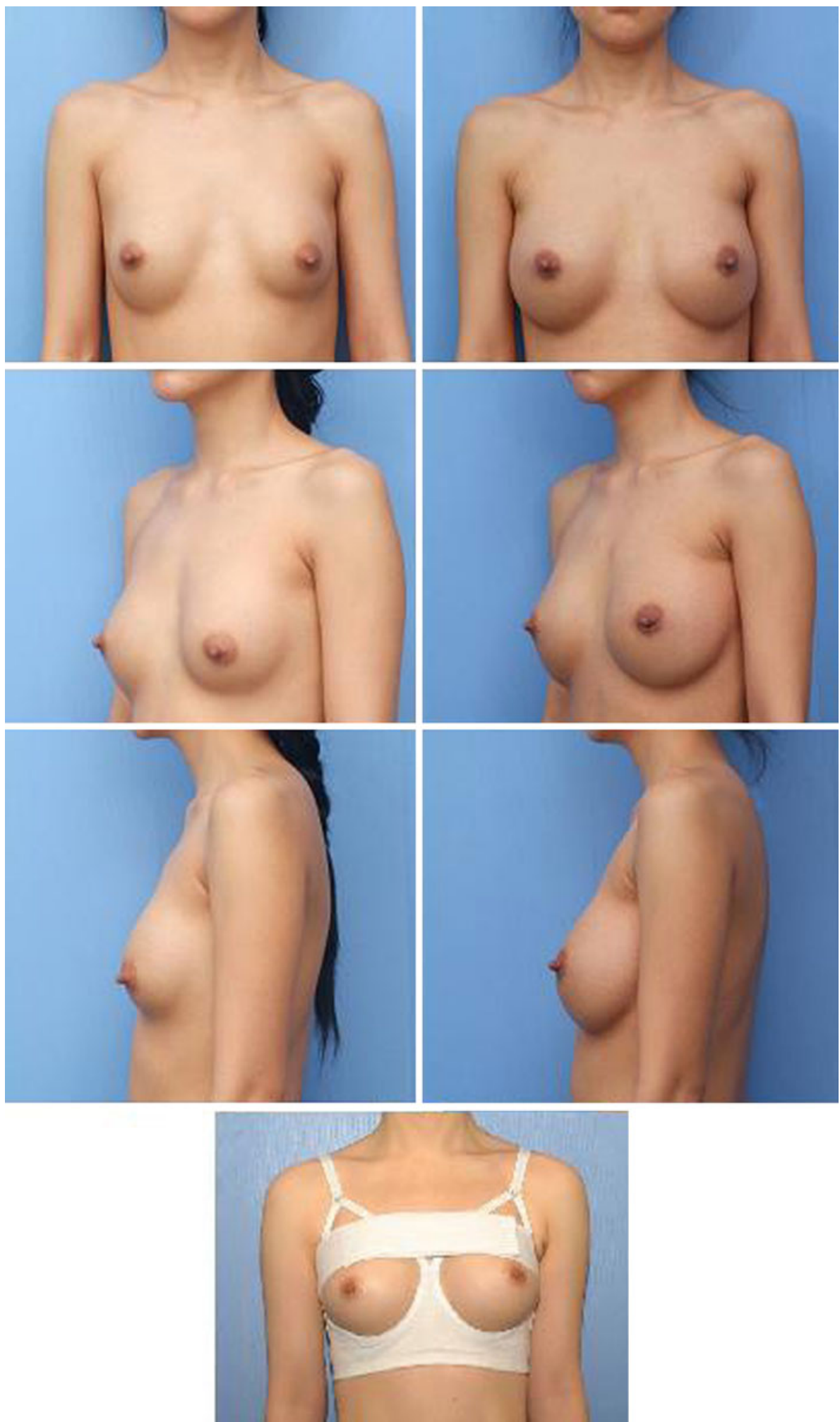

was dissected away from the pectoralis major muscle and a new space was created behind the muscle and anterior to the capsule; a new pocket was created so that an implant may be placed there. In cases of infected capsule, calcified capsule, or ruptured capsule where silicone gel was released, it is undesirable to retain the capsule. In these circumstances, a total capsulectomy is preferable [4, 13].

As described, postoperative recurrence of capsular contractures can be reduced with this technique because the implant does not come in contact with the inner surface of 
Fig. 3 A 33 year-old woman. Left column 3 years later after transaxillary subpectoral breast augmentation. Note the upward migration of the bilateral breast due to capsular contracture.

Right column postoperative 6 month view

Fig. 4 Left A 33-year-old woman with symmastia after original breast augmentation. Right Postoperative 9-month view
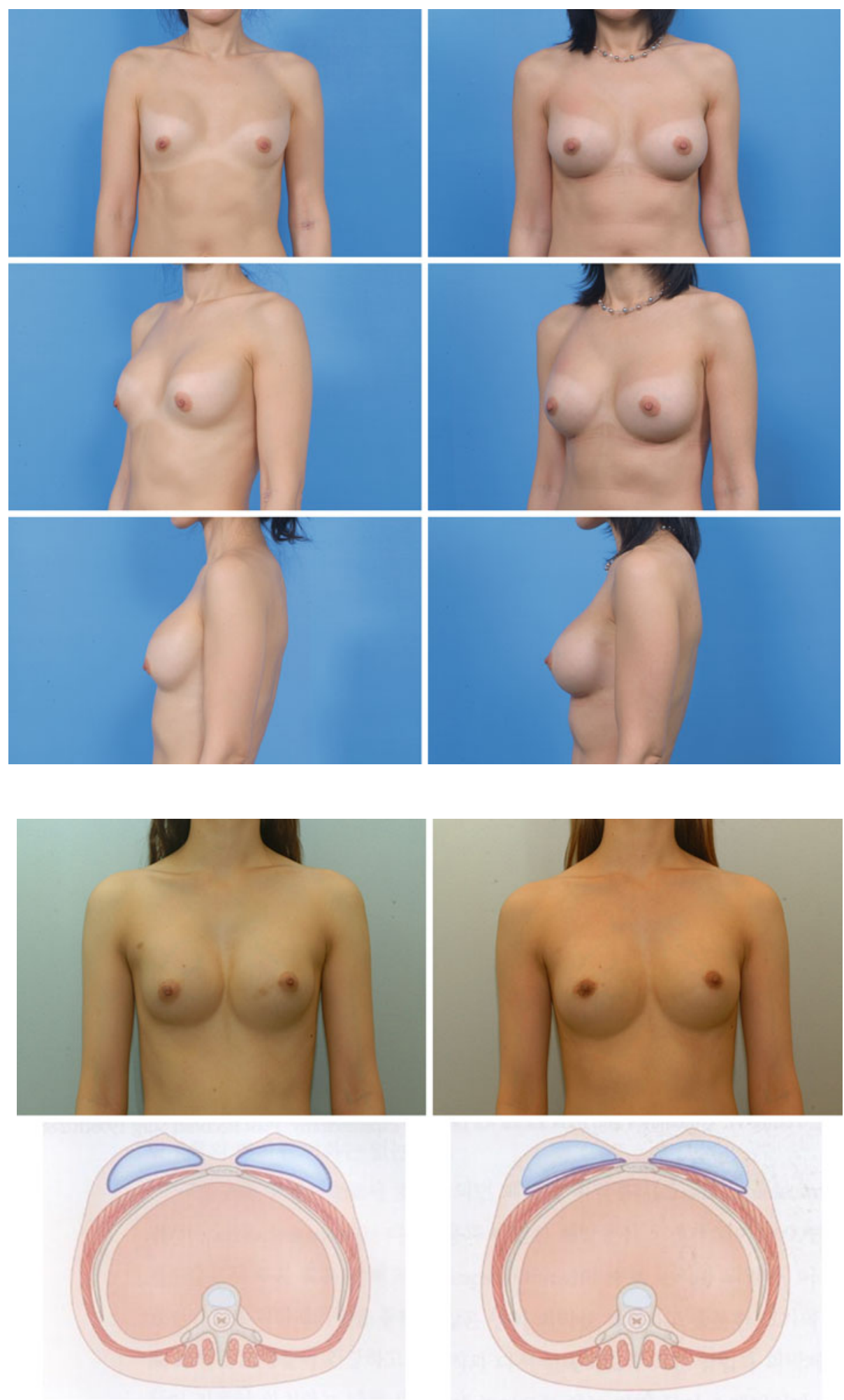

the capsule. Moreover, the shape and size of the new pocket can be formed as desired by the patient, similar to the initial operation. However, there is some controversy regarding how the retained capsule may change over time. Hardt et al. [14] reported that retained implant capsules may evolve to take several forms, including a speculated mass similar to a breast carcinoma, dense calcifications that obscure the neighboring breast tissue on imaging studies, or a cystic mass due to persistent serous effusion, expansile hematoma, or encapsulated silicone-filled cysts. However, 
Rockwell et al. [4] described retained capsules that were still present after silicone gel implant removal. Cihat et al. [15] reported that retained capsules would most likely disappear in time if the capsule is not stimulated by the implant. We also believe that retained capsules will be resorbed or thinned over time. Consequently, we need more long-term follow-up studies with radiological and histological surveys [16].

Leukotriene antagonists are known to effectively prevent capsular contractures [17]. Zafirlukast (Accolate ${ }^{\circledR}$, AstraZeneca, Wilmington, DE) was prescribed to all patients postoperatively since October 2002, except for patients with hepatic dysfunction (due to its association with hepatic failure). No signs of hepatotoxic effects were observed following the leukotriene antagonist therapy.

\section{Conclusion}

The described technique in this report makes possible a secondary operation that reduces capsular contracture through a scar tissue-free environment, unlike a partial capsulectomy or capsulotomy. Furthermore, the dissection of the capsule is made significantly easier than in cases of total capsulectomy. We feel that further prospective studies on a larger scale are necessary to examine this technique further and hope that this report will contribute to the treatment of this feared complication of a common procedure.

Conflict of interest H. K. Lee, U. Jin, and Y. Lee have no conflicts of interest or financial ties to disclose.

Open Access This article is distributed under the terms of the Creative Commons Attribution Noncommercial License which permits any noncommercial use, distribution, and reproduction in any medium, provided the original author(s) and source are credited.

\section{References}

1. Tebbetts JB (2006) Achieving a zero percent reoperation rate at 3 years in a 50-consecutive-case augmentation mammaplasty premarket approval study. Plast Reconstr Surg 118:1453-1457
2. Collis N, Sharpe DT (2000) Recurrence of subglandular breast implant capsular contracture: anterior versus total capsulectomy. Plast Reconstr Surg 106:792-797

3. Chasen PE (2005) Breast capsulorrhaphy revisited: a simple technique for complex problem. Plast Reconstr Surg 115:296-301; discussion 302-303

4. Rockwell WB, Casey HD, Cheng CA (1998) Breast capsule persistence after breast implant removal. Plast Reconstr Surg 101:1085-1088

5. Tebbetts JB (2001) Dual plane breast augmentation: optimizing implant-soft-tissue relationships in a wide range of breast types. Plast Reconstr Surg 107:1255-1272

6. Schlesinger LS, Ellenbogen Richard, Desvigne MN, Svehlak S, Heck R (2002) Zafirlukast (Accolate): a new treatment for capsular contracture. Aesthetic Surg J 22:329-336

7. Becker H, Springer R (1999) Prevention of capsular contracture. Plast Reconstr Surg 103:1766-1768; discussion 1769-1774

8. Camirand A, Doucet J (2000) Breast augmentation: teaching our patients how compression can help prevent capsular contracture. Aesthetic Plast Surg 24:221-226

9. Baker JL Jr, Bartels RJ, Douglas WM (1976) Closed compression technique for rupturing a contracted capsule around a breast implant. Plast Reconstr Surg 58:137-141

10. Nelson GD (1980) Complications of closed compression after augmentation mammaplasty. Plast Reconstr Surg 66:71-73

11. Moufarrege R, Beauregard G, Bosse JP, Papillon J, Perras C (1987) Outcome of mammary capsulotomies. Ann Plast Surg 19:62-64

12. Tamboto H, Vickery K, Deva AK (2010) Subclinical (biofilm) infection causes capsular contracture in a porcine model following augmentation mammaplasty. Plast Reconstr Surg 126: 835-842

13. Young VL (1998) Guidelines and indications for breast implant capsulectomy. Plast Reconstr Surg 102:884-891; discussion 892-894

14. Hardt NS, Yu L, LaTorre G, Steinbach B (1995) Complications related to retained breast implant capsules. Plast Reconstr Surg 95:364-371

15. Baran CN, Peker F, Ortak T, Sensoz O, Baran NK (2001) A different strategy in the surgical treatment of capsular contracture: leave capsule intact. Aesthetic Plast Surg 25:427-431

16. Maxwell GP, Gabriel A (2008) The neopectoral pocket in revisionary breast surgery. Aesthetic Surg J 28:463-467

17. Scuderi N, Mazzocchi M, Fioramonti P, Bistoni G (2006) The effects of zafirlukast on capsular contracture: preliminary report. Aesthetic Plast Surg 30:513-530 